Original
article

Department of Epidemiology and Biostatistics, Institute of Experimental and Clinical Medicine, Tallinn, Estonia M Kibur

Microbiology and Tumor Biology Centre, Karolinska Institute, Stockholm, Sweden

$\mathrm{V}$ af Geijerstamm Z Wang

J Dillner

Finnish Cancer Registry, Helsinki, Finland

E Pukkala

T Luostarinen

University of Helsinki, Helsinki, Finland

J Paavonen

Department of Infectious Disease Epidemiology, and Department in Oulu, National Public Health Institute, Helsinki and Oulu, Finland

P Koskela

M Lehtinen

University of Tampere, School of Public

Health, Tampere,

Finland

J Dillner

M Kibur

M Lehtinen

Laboratory of Cellular Oncology, National

Cancer Institute, Bethesda, MD, USA J Schiller

Correspondence to: Dr M Lehtinen, Department of Infectious Disease Epidemiology, NPHI, Mannerheimintie 166, 00300, Helsinki, Finland

\title{
Attack rates of human papillomavirus type 16 and cervical neoplasia in primiparous women and field trial designs for HPV16 vaccination
}

M Kibur, V af Geijerstamm, E Pukkala, P Koskela, T Luostarinen, J Paavonen, J Schiller, $\mathrm{Z}$ Wang, J Dillner, M Lehtinen

Background: Identification of human papillomavirus type 16 (HPV16) as the major risk factor for cervical neoplasia, and mass production of DNA free HPV capsids have paved the way to preventive vaccination trials. Design of such trials requires reliable attack rate data.

Objective: Determination of (1) HPV16 and (2) cervical neoplasia attack rates in primiparous women. Estimation of actuarial sample sizes for HPV16 vaccination phase IV trials.

Design: A longitudinal cohort study.

Methods: Population based Finnish Maternity Cohort (FMC) and Finnish Cancer Registry (FCR) were linked for the identification of two cohorts of primiparous women: (1) a random subsample of the FMC: 1656 women with two pregnancies between 1983-9 or 1990-6 and living in the Helsinki metropolitan area, and (2) all 72791 primiparous women living in the same area during 1983-94. Attack rate for persistent HPV16 infection (1) was estimated in 1279 seronegative women by proportion of seroconversions between the first and the second pregnancy. Comparable 10 year cumulative incidence rate (CR) of cervical intraepithelial neoplasia grade III and cervical cancer (CIN III+) (2) was estimated based on cases registered at the FCR during 1991-4.

Results: The HPV16 attack rates were $13.8 \%$ (<18 years), 7.0\% (18-19 years), 2.3\% (21 years), $2.4 \%$ (23 years), and $4.5 \%$ ( $<25$ years). Number of vaccinees required for a 5 year efficacy trial with persistent HPV16 infection as the end point ranged between 1000 and 3900, assuming $80 \%$ power, $90 \%-70 \%$ vaccine efficacy (VE), and misclassification. The CRs of CIN III+ were $0.33 \%$ (<18 years), $0.44 \%$ ( $18-19$ years), $0.21 \%$ (20-24 years), and $0.28 \%$ ( $<25$ years). Number of vaccinees required for a 10 year efficacy trial with HPV16 positive CIN III+ as the end point was 15000 assuming $80 \%$ power, $90 \% \mathrm{VE}$, and $75 \%$ aetiological fraction of CIN III+ for HPV16.

Conclusions: The attack rates of HPV16 and CIN III+ identify primiparous women under 25 years of age among target populations for postnatal HPV vaccination at phase II/III trials.

(Sex Transm Inf 2000;76:13-17)

Keywords: attack rate; cervical neoplasia; human papillomavirus; vaccination

\section{Introduction}

Epidemiological studies have found human papillomavirus type 16 (HPV16) to be the major aetiological factor for cervical intraepithelial neoplasia (CIN) grade III and cervical cancer. ${ }^{2}$ HPV16 and other HPV DNA is found in more than $90 \%$ of the tumours. ${ }^{1}$ Infection with HPV 16 is associated with an increased risk for the subsequent development of squamous intraepithelial lesions, ${ }^{3}$ cervical cancer, ${ }^{4}$ and other anogenital cancers. ${ }^{6}$ This has stimulated development of preventive vaccines against the virus to control cervical cancer, a common cancer in women worldwide. ${ }^{7}$ Production of empty capsids or virus-like particles (VLPs), in different expression vector systems was a

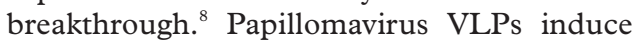
protection against experimental infection and tumour development in rabbits, dogs, and cows. $^{9-12}$

A vaccine against another human tumour virus, hepatitis B virus (HBV), is based on the same principle. ${ }^{13}$ This vaccine has greatly reduced incidence of hepatitis $B$ infection and number of chronic viral carriers in high endemic areas. ${ }^{14}{ }^{15}$ The exclusive sexual transmission of HPV infection makes it an attractive target for vaccination since even low efficacy vaccines to STDs have a substantial effect at the population level provided wide coverage of the vaccination programme is guaranteed. ${ }^{16}$

Annually 65000 pregnant Finnish women participate in screening for congenital infections ${ }^{17}$ with an option for postnatal immunisation. Of the primiparous women, about $50 \%$ become pregnant again/are rescreened within 5 years thus forming Finnish Maternity Cohort (FMC) of the National Public Health Institute (NPHI). Population based registration of cervical neoplasia by the Finnish Cancer Registry (FCR) was established in the 1950 s and has $100 \%$ coverage. $^{18}$ We exploited linkage of the FMC and FCR data files for the determination HPV16 and cervical neoplasia attack rates in primiparous women. 
Table 1 HPV16 seroprevalence at the first pregnancy, number of seroconversions, and attack rates in age stratified primiparous women resident in the Helsinki metropolitan area during 1983-9 (A) and 1990-6 (B)

\begin{tabular}{lclcl}
\hline $\begin{array}{l}\text { Age group } \\
\text { (years) }\end{array}$ & No of women & $\begin{array}{l}\text { No (\%) of } \\
\text { seropositives }\end{array}$ & No of seroconversions & $\begin{array}{l}\text { Attack rate } \\
\text { (\%) }\end{array}$ \\
\hline A & & & & \\
$\leqslant 17$ & 46 & $13(28.3)$ & 3 & 9.1 \\
$18-19$ & 186 & $52(28.0)$ & 10 & 7.5 \\
21 & 153 & $36(23.5)$ & 3 & 3.6 \\
23 & 183 & $31(16.9)$ & 6 & -1.5 \\
25 & 92 & $18(19.6)$ & - & \\
30 & 90 & $25(27.8)$ & 1 & 15.2 \\
& & & & 6.6 \\
B & 139 & $27(19.4)$ & 17 & 2.1 \\
$\leqslant 17$ & 196 & $44(22.5)$ & 10 & 0.7 \\
$18-19$ & 188 & $45(23.9)$ & 3 & 2.5 \\
21 & 191 & $49(25.7)$ & 1 & 1.5 \\
23 & 97 & $17(17.5)$ & 2 & \\
25 & 95 & $30(31.6)$ & 1 & \\
30 & & & & \\
\hline
\end{tabular}

\section{Subjects and methods}

SUBJECTS

The FMC receives, analyses, and stores serum samples from almost all (98\%) pregnant women in Finland. The samples are drawn at maternity clinics during the first trimester of pregnancy and analysed at the NPHI. Sample storage to $-20^{\circ} \mathrm{C}$ started in 1983-4 and is still ongoing. ${ }^{17}$ The FCM serum bank now contains more than one million samples donated by 460000 women.

We identified in the FMC a subcohort of women, living in the Helsinki metropolitan area, who became pregnant for the first time in 1983-4 or in 1990-1 and again within a maximum of 5 years. A subsample of every second birth cohort $<25$ years of age at the first pregnancy, and comparable 30 year old women were chosen for the study. However, serum samples for all available women in the age categories $\leqslant 17$ and 21 years (1983-4) were retrieved to have representative sample sizes also for these age groups. Overall, 1260 primiparous women from 1983-4 and 3432 primiparous women from 1990-1 were available for the study. Two hundred women in the age groups 19, 21, and 23 years, and 100 women in the age groups of 25 and 30 years were randomly chosen for the determination of HPV16 antibodies. For the age group $\leqslant 17$ years $58(1983-4)$ and 148 (1990-1) women, and for the age group 21 years 173 (1983-4) women were available. After sample retrieval paired sera (mean interval 31.3 months) were available for 1656 women

Another subcohort identified within the FMC comprised all the 72791 primiparous women living in the Helsinki metropolitan area between 1983-94. Since the first pregnancy these women were followed up to the end of 1994 to identify new cases of CIN III+ (CIN III and cervical cancer) reported to the FCR between 1991-4. In Finland the registration of cervical cancer, carcinoma in situ, and CIN III (comprising dysplasia gravis and carcinoma in situ) has been in operation since 1953, 1961 , and 1991, respectively. About $95 \%$ of the diagnoses are histologically confirmed. ${ }^{18}$
During 1991-4 the following age stratas: $\leqslant 17,18-19,20-24,25-29$, and $\geqslant 30$ years consisting of $14027,9092,28852,14732$, and 6088 women, comprised 29 049, 19 149, 59966,30112 , and 12387 person years for the first 5 years of follow up, and 14275 , 9151, 27 550, 13718 , and 5985 person years for the next 5 years of the follow up, respectively.

LABORATORY ANALYSIS

HPV16 IgG antibody analysis was done by a standard ELISA using ultracentrifugation purified baculovirus expressed HPV16 VLPs comprising both the $\mathrm{L}_{1}$ and $\mathrm{L}_{2}$ proteins. ${ }^{19}{ }^{20}$ An optical density of 0.100 , previously shown to discriminate HPV16 infected, sexually experienced women from sexually inexperienced women ${ }^{21}$ was used as the cut off level.

STATISTICAL ANALYSES

HPV16 attack rate was defined as the proportion of HPV16 seroconversions between serum sampling at approximately 12 weeks of gestation of the first and the second pregnancy among those seronegative at the first pregnancy. For women under 25 years of age, age adjusted HPV16 attack rate was estimated using the numbers of corresponding primiparous women resident in the Helsinki metropolitan area in 1990-1 as weights. Overall HPV16 seroprevalence at the first pregnancy was similarly estimated as weighted average of age specific seroprevalences.

CIN III+ attack rate was defined as the 10 year cumulative incidence rate (CR) of cases diagnosed during 1991-4. For women under 25 years of age, age adjusted annual incidence for the first and the last 5 years and the CR of CIN III+ were estimated as weighted averages of the age specific rates using the age specific numbers of corresponding person years as weights.

Trends of the attack rates by age were evaluated using the Cochran-Armitage trend test by STATXAT-3 (Cytel Software Corporation, Cambridge, MA, USA).

Numbers of vaccinees required for a $1: 1$ placebo controlled efficacy trial were estimated with $80 \%$ power $(\mathrm{p}=0.05)$ using the observed HPV16 and CIN III+ attack rates, and assuming different levels of vaccine efficacy (VE). ${ }^{22}$ The estimates were calculated using a publicly available statistical software (Public Health Laboratory Service, Colindale).

\section{Results}

In 1983-4 and 1990-1 the age specific HPV16 seroprevalence among primiparous women varied between $17-28 \%$ and $18-32 \%$ (table 1 ). The age adjusted HPV16 seroprevalence was $24 \%$ for both time periods. Among the seronegatives, the combined HPV16 attack rates varied between 13.8 and 1.3 , and showed statistically an extremely significant decrease by increasing age at 
Table 2 Number of primiparous women required for a double blinded postnatal efficacy trial on preventive HPV16 vaccination with the second pregnancy as the end point

\begin{tabular}{llll}
\hline & & \multicolumn{2}{l}{$\begin{array}{l}\text { Number of vaccinees at a } \\
\text { given vaccine efficacy }\end{array}$} \\
\cline { 3 - 4 } $\begin{array}{l}\text { Age group } \\
\text { (years) }\end{array}$ & $\begin{array}{l}\text { HPV16 attack } \\
\text { rate }\end{array}$ & $90 \%$ & $70 \%$ \\
\hline$\leqslant 17$ & 13.8 & 142 & 274 \\
$18-19$ & 7.0 & 292 & 566 \\
21 & 2.3 & 914 & 1782 \\
23 & 2.4 & 876 & 1706 \\
25 & 1.3 & 1626 & 3176 \\
$<25$ & 4.5 & 460 & 898 \\
\hline
\end{tabular}

${ }^{\star}$ Between the first and the second pregnancy.

the first pregnancy $\mathrm{p}<0.0001$. The age adjusted HPV16 attack rate for primiparous women under 25 years of age was $4.5 \%$ (table 2).

Based on the observed attack rates we estimated the number of women required for a double blinded efficacy trial to protect against HPV16 infection with a power of $80 \%$ and assuming $\mathrm{VE}$ of $90 \%$ or $70 \%$. The estimated numbers of vaccinees varied between $142 \quad(90 \%$ VE, first pregnancy $\leqslant 17$ years) and 3176 (70\% VE, first pregnancy at 25 years). With the observed distribution of primiparous women under 25 years of age, and no losses to follow up 460-898 primiparous women are required (table 2).

The CIN III+ rates of primiparous women were calculated after linkage of the FMC and FCR data files with 221342 person years during 10 years of follow up. The annual age specific incidence rates of CIN III+ varied between 56.5 (18-19 years) and 18.3 (25-29 years). For women under 25 years of age the age adjusted rate was 38.0. The CRs varied between $0.44 \%$ (18-19 years) and $0.12 \%$ (25-29 years). For women under 25 years of age the cumulative rate was $0.28 \%$ (table 3).

With the observed CRs, a power of $80 \%$ and VE of $90-70 \%$ against CIN III+ the number of women required for a double blinded long term efficacy trial varied between 4800 20000 (table 4). Assuming 75\% HPV16 positivity of CIN III+ and a VE of $90 \%$, the estimated size of a trial involving HPV16 negative women who are, or will be, pregnant would be 15000 .

Table 3 Incidence $\left(I / 10^{5}\right)$ and 10 year cumulative incidence rate (CR) of cervical intraepithelial neoplasia grade III and invasive cervical cancer in primiparous women resident in the Helsinki metropolitan area during 1983-94

\begin{tabular}{|c|c|c|c|c|c|}
\hline \multirow{3}{*}{$\begin{array}{l}\text { Age group } \\
\text { (years) }\end{array}$} & \multirow[b]{3}{*}{ No of women } & \multicolumn{4}{|c|}{ Follow up time (years) } \\
\hline & & $0-5$ & $6-10$ & $0-10$ & $C R$ \\
\hline & & $1 / 10^{5}$ & $I / 10^{5}$ & $I / 10^{5}$ & $\%$ \\
\hline$\leqslant 17$ & 14027 & 31.0 & 70.1 & 43.9 & 0.33 \\
\hline $18-19$ & 9092 & 52.2 & 65.6 & 56.5 & 0.44 \\
\hline $20-24$ & 28852 & 28.3 & 32.7 & 29.7 & 0.21 \\
\hline $25-29$ & 14732 & 23.3 & 7.3 & 18.3 & 0.12 \\
\hline 30 & 6088 & 32.2 & - & 21.8 & 0.16 \\
\hline$<25$ & 51971 & 33.1 & 48.1 & 38.0 & 0.28 \\
\hline
\end{tabular}

Table 4 Number of women required for a double blinded efficacy trial on preventive HPV16 vaccination with cervical intraepithelial neoplasia grade III and invasive cervical cancer as the end point

\begin{tabular}{lllr}
\hline \multirow{2}{*}{$\begin{array}{l}\text { Age group } \\
\text { (years) }\end{array}$} & $\begin{array}{l}\text { CIN III+ attack } \\
\text { rate \% }\end{array}$ & \multicolumn{2}{c}{ Number of vaccinees at a given VE } \\
\cline { 3 - 4 } & & $90 \%$ & $70 \%$ \\
\hline 18 & 0.33 & 6446 & 12592 \\
$18-19$ & 0.44 & 4832 & 9436 \\
$20-24$ & 0.21 & 10138 & 19804 \\
$<25$ & 0.28 & 7600 & 14846 \\
\hline
\end{tabular}

\section{Discussion}

No major differences in the age specific HPV16 prevalence or incidence were found between the 1980s and 1990s among primiparous women in the Helsinki metropolitan area. The HPV16 attack rate between samples taken at the first and the second pregnancy (that is, within an average of 2.5 years) for women under 25 years of age was $4.5 \%$. Annual HPV incidence in Finnish women of similar age by cytology is $7 \% .^{23}$ This is in accordance with our average attack rate assuming that HPV16 accounts for about one third of cytologically detectable HPV infections, and does not suggest major differences between the pregnant and general populations of young women. The HPV16 attack rates rapidly decreased with increasing age at the first pregnancy, although the mean time between the two pregnancies remained comparable (data not shown). This is also in accordance with the PCR studies that show a peak of all genital HPV and HPV16 infections between 20-24 years of age. ${ }^{24} 25$

HPV VLP serology has been established in numerous studies ${ }^{19-21} 26-31$ and shows good interlaboratory agreement. ${ }^{31}$ Persistence of HPV16 IgG antibodies in our material has been assessed in detail elsewhere ${ }^{26}$; briefly, loss of HPV16 antibodies within the 5 year follow up time was extremely rare $0.5 \%$. Compared with polymerase chain reaction (PCR) the sensitivity/ specificity of HPV16 serology range from 50\%/ $95 \%$ to $99 \% / 90 \%{ }^{19} 2027$ depending mostly on the study material - for example, population, type of lesions, prevalent versus incident infections. According to our experience the median time from HPV16 acquisition to detectable antibody response is 4 rather than 8 months, ${ }^{21} 273233$ which suggests that only a minor proportion of the infections we are identifying by seroconversion may have taken place before the pregnancy. After correction for misclassification, ${ }^{34}$ the HPV16 seroprevalence estimates ranged between $17 \%$ and $47 \%$. Comparable considerations for the HPV16 attack rate among women under 25 years of age yielded a range of $3.2 \%$ to $8.8 \%$. Thus, about every fifth HPV16 infection by the second pregnancy occurs between the first and the second pregnancy.

Persistent HPV infection is considered a prerequisite for development of an HPV associated cancer. ${ }^{1}$ Detection of viral DNA at the cervix by PCR has the advantage of a higher sensitivity but without serial sampling will also detect short lived, transient infections. By limiting our study to seronegative women and using seroconversion, a marker that primarily detects persistent HPV16 infections s--30 $^{35}$ we were able to estimate the attack rate of biologically relevant infections 
among susceptible women under the closest possible surveillance-that is, in between two pregnancies. Considering the misclassification, and the fact that $50 \%$ of the women will be pregnant again within the next 5 years, but that practically none $(<2 \%)$ of these women will be lost to follow up, 1000 and 3500 are safe estimates of minimum and maximum numbers of vaccinees required for a 5 year efficacy trial with persistent HPV16 infection as the end point.

During the early 1990s the annual incidence of CIN III+ in young primiparous women followed for 6-10 years was relatively high (48.1/ $100000)$. This probably reflects the high HPV16 attack rates in the corresponding age cohorts 6-10 years earlier. The first round of the organised mass screening for cervical neoplasia in the Helsinki metropolitan area takes place at the age of 25 years. Since the 1970s the relatively constant protective effect of organised mass screening rounds on the CIN III+ incidence ${ }^{36}$ was clearly visible in the oldest age cohort with first pregnancy $\geqslant 30$ years of age. No CIN III+ cases were found between 6 to 10 years of follow up in these women. Considering future vaccination trials the effect of organised screening is, however, possible to control for by randomisation whereas the effect of opportunistic screening that dominates in many developed countries cannot be controlled.

For design of HPV vaccination trials, the choice of time of vaccination and end points is critical. Practically all women who would benefit from preventive HPV vaccination are susceptible to both infection with the genital HPVs and pregnancy. The least likely time period for a woman to be pregnant is the 3 months after delivery. This favours postnatal vaccination in large scale public health trials. Use of HPV16 infection defined by PCR or CIN I as the end point is mostly of interest to investigate vaccine efficacy against the infection itself, and could probably be accomplished as scheduled above.

Reliable evidence of a cancer protective effect of preventive HPV vaccination can, however, only come from studies using an immediate precursor of cervical cancer, such as CIN III, or cervical cancer as the end point. The Finnish Cancer Registry receives systematic quality controlled information on all cases of CIN III+ diagnosed in Finland ${ }^{18}$ precluding losses to any follow up period. Assuming that $65 \%$ to $75 \%$ of CIN III+ lesions in Finland are HPV16 positive ${ }^{37} 15000$ is the best available estimate of the number of HPV16 negative women under 25 years of age required for a long term trial with CIN III+ as the end point. As $65 \%$ to $90 \%$ of women who will acquire HPV16 infection (as determined by seropositivity) by their second pregnancy had acquired it by week 12 of their first pregnancy primiparous women would not be sufficient target group for a phase IV vaccine trial where such high numbers of subjects was required. Enrolment of large numbers of adolescents at different subsidiaries of the organised health care would be required for such a study.

The excellent technical assistance of Ms Carina Eklund is gratefully acknowledged. This work was supported by grants from the Finnish Cancer Organisations, Academy of Finland, Nordic Academy for Advanced Study, and from the Swedish Cancer Society. 1 IARC. Human papillomaviruses. IARC monographs on
assessment of carcinogenic risks to humans. Vol 64. Lyons: assessment of carcinogenic risks to hum
IARC Scientific Publications, 1995.

2 Schiffmann MH. New epidemiology of human papillomavirus infection and cervical neoplasia. $F$ Natl Cancer Inst 1995;87:1345-7.

3 Ho GYF, Bierman R, Beardsley L, et al. Natural history of cervicovaginal papillomavirus infection in young women. $N$ Engl f Med 1998;338:423-8.

4 Lehtinen M, Dillner J, Knekt P, et al. Serologically diagnosed infection with human papillomavirus type 16 and risk for subsequent development of cervical carcinoma: nested case-control study. BMf 1996;312:537-9.

5 Dillner J, Lehtinen M, Bjorge T, et al. Prospective seroepidemiologic study of human papillomavirus infection as a risk factor for invasive cervical cancer. F Natl Cantion as a risk factor for in
cer Inst 1997;89:1293-9.

6 Bjorge T, Dillner J, Anttila T, et al. Prospective seroepidemiological study on human papillomavirus type 16,18 and 33 and non-cervical anogenital cancers. BMF 1997;315: 646-9.

7 Parkin DM, Pisani P, Ferlay J. Estimates of the worldwide incidence of eighteen major cancers in 1985. Int 7 Cancer 1993; 54:594-606.

8 Frazer IH. The role of vaccines in the control of STDs:HPV vaccines. Genitourin Med 1996;72:398-403.

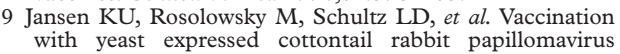
with yeast expressed cottontail rabbit papillomavirus
(CRPV) virus-like particles protects rabbits from CRPV(CRPV) virus-like particles protects rabbits from CRP

10 Breitburd F, Kirnbauer R, Hubbert NL, et al. Immunization with virus-like particles from cottontail rabbit papillomavirus (CRPV) can protect against experimental CRPV infection. F Virol 1995;69:3959-63.

11 Kirnbauer R, Chandrachud LM, O'Neil BW, et al. Virus-like particles of bovine papillomavirus type 4 in prophylactic and therapeutic immunization. Virology 1996; 219:37-44.

12 Suzich JA, Ghim S-J, Palmer-Hill F, et al. Systemic immunization with papillomavirus L1 protein completely prevents zation with papillomavirus L1 protein completely prevents
the development of viral mucosal papillomas. Proc Natl the development of viral mucosal

13 Petre J, van Wijnendaele F, De Neis B, et al. Development of hepatitis B vaccine from transformed yeast cells. Postgraduate Med $\mathcal{F}$ 1987;63:73-81

14 Whittle HC, Maine N, Pilkington J, et al. Long-term efficacy of continuing hepatitis B vaccination in infancy in two Gambian villages. Lancet 1995;345:1089-92.

15 Wainwright RB, Bulkow LR, Parkinson AJ, et al. Protection provided by hepatitis B vaccine in a Yupik Eskimo population-results of 10-year study. F Infect Dis 1997;175: $674-7$.

16 Andersson RM, Garnett GP. Low-efficacy HIV vaccines: potential for community-based intervention programmes. Lancet 1996;348:1010-3.

17 Tuomilehto J, Zimet P, Mackay IR, et al. Antibodies to glutamic acid decarboxylase as predictors of insulindependent diabetes mellitus before clinical onset of disease. Lancet 1994;343:1383-5.

18 Teppo L, Pukkala E, Lehtonen M. Data quality and quality control of a population-based cancer registry. Experience in Finland. Acta Oncol 1994;33:365-9.

19 Kirnbauer R, Hubbert NL, Wheeler CM, et al. virus-like particle enzyme-linked immunosorbent assay detects serum antibodies in majority of women infected with human papillomavirus type 16. F Natl Cancer Inst 1994; 86:494-8.

20 Dillner J, Wiklund F, Lenner P, et al. Antibodies against linear and conformational epitopes of human papillomavirus type 16 that independently associate with incident cervical cancer. Int $\mathcal{F}$ Cancer 1995;60:377-82.

21 Andersson-Ellström A, Dillner J, Hagmar B, et al. Comparison of development of serum antibodies to HPV16 and HPV 33 and acquisition of cervical HPV DNA among exually experienced and virginal young girls. A longitudinal cohort study. Sex Transm Dis 1996;23:234-8.

22 Neill R. On sample size to estimate the protective efficacy of a vaccine. Stat Med 1988;7:1279-88.

23 Syrjänen K, Hakama M, Saarikoski S, et al. Prevalence, incidence and estimated life-time risk of cervical human papillomavirus infection in a non-selected Finnish female population. Sex Transm Dis 1990;17:15-9.

24 Melkert PWJ, Hopman E, van der Brule JC, et al. Prevalence of HPV in cytomorphologically normal cervical smears, as determined by the polymerase chain reaction, is agedetermined by the polymerase chain reac

25 Schiffmann MH. Epidemiology of cervical human papillomavirus infection. Curr Topics Microbiol Immunol 1994;196: 55-81.

26 Af Geijerstamm V, Kibur M, Wang Z, et al. Stability over time of serum antibody levels to human papillomavirus type 16. F Infect Dis 1998;177:1710-4.

27 Carter JJ, Koutsky LA, Wipf GC, et al. The natural history of human papillomavirus type 16 capsid antibodies among a cohort of university women. F Infect Dis 1996;174:927-6.

28 Dillner J, Kallings I, Brihmer C, et al. Seropositivities to human papillomavirus types 16,18 or 33 capsids and to
Chlamydia trachomatis are markers of sexual behaviour. $f$ Infect Dis 1996;173:1394-8.

29 Wideroff L, Schiffman MH, Nonnenmacher B, et al. Evaluation of seroreactivity to human papillomavirus type 16 virus-like particles in an incident case-control study of cervical neoplasia. F Infect Dis 1995;172:1425-30. 
30 Wideroff L, Schifman MH, Hoover RE, et al. Epidemiologic determinants of seroreactivity to human papillomavirus ervical HPV-16 DNA-positive and -negative women. F Infect Dis 1996;174 937-43.

31 Strickler HD, Hildesheim A, Viscidi RP, et al. Interlaboratory aggreement among results of human papillomavirus type 16 enzyme-linked immunosorbent assays. F Clin Microbiol 1997;35:1751-6.

32 Wikström A, van Doornum GJJ, Quint WGV, et al. Identification of human papillomavirus seroconversions. $\mathcal{F}$ Gen Virol 1995;76:529-39.

33 Wikström A, van Doornum GJJ, Kirnbauer R, et al. Prospective study on the development of antibodies against human papillomavirus type 6 among patients with condylomata acuminata or new asymptomatic infection. $f$ Med Virol 1995;46:368-74.
34 Franco EL. Measurement errors in epidemiological studies of human papillomavirus and cervical cancer. In: Munoz , Bosch FX, Shah KV, et al, eds. The epidemiology of cervical cancer and human papillomavirus. Lyons: IARC Scien-

35 De Gruijl TD, Bontkes HJ, Walboomers JMM, et al. Immunoglobulin $G$ responses against human papillomavirus type 16 virus like particles in a prospective non-intervention cohort study of women with cervical intraepithelial neoplasia. F Natl Cancer Inst 1997;89:630-8.

36 Läärä $E$, Day NE, Hakama $M$. Trends in mortality from cervical cancer in the Nordic countries:association with organised screening programmes. Lancet 1987;i:1247-9.

37 Iwasawa A, Nieminen P, Lehtinen $M$, et al. Human papillomavirus DNA in uterine cervix squamous cell carcinoma and adenocarcinoma detected by polymerase chain reaction. Cancer 1996;77:2275-9.

\title{
Joint Meeting of MSSVD and the ASTDA
}

\author{
3-7 May 2000, Baltimore Marriott Inner Harbor Hotel, Baltimore, Maryland, USA
}

To mark the unique nature of the millennial year, for the first time in its 78 year history the Medical Society for the Study of Venereal Diseases will hold its Spring Meeting jointly with the American Sexually Transmitted Disease Association (President, Professor Julius Schacter). Our local host will be Professor Jonathan Zenilman of Johns Hopkins Medical School. The scientific programme will consist of plenary lectures and round table discussions, delivered by world authorities. There will also be oral and poster presentations of original work.

Further mailings will follow to MSSVD and ASTDA members. People who do not belong to either of these organisations and who would like to receive further information should contact: Dr Keith Radcliffe, Honorary Assistant Secretary, MSSVD (fax: +44 (0) 121-237 5729; email: k.w.radcliffe@bham.ac.uk). 